Notre Dame Journal of Formal Logic

Volume IV, Number 2, April 1963

\title{
ON DIFFERENCES OF CERTAIN STRUCTURED SETS
}

\author{
ALBERT A. MULLIN
}

The author dedicates this paper to the memory of Evariste Galois on the occasion of the 150 th anniversary of that mathematician's birthday, 25 October 1811.

Introduction: The activities of modern algebra are in accord with the traditions of the scientific method in that they stress the similarities (e.g., isomorphisms) of various canonic algebraic structures (e.g., groups and rings) thereby largely ignoring, for eminently practical reasons, differences between algebraic structures. This paper commences, in a modest way, some investigations into differences between certain structured sets. Such inquiries can only be a supplementary testament to the abundance of fine works performed by the many researchers in studying similarities between algebraic structures. Indeed without those earlier works to draw upon, the present kind of study would be impossible. It is, also, a tribute to the scientific method that such an investigation as the present one is quite natural, and not the product of an attempt to produce novelty, which is a poor reason to recommend any inquiry.

Definitions: As is well known one structured set is similar to a second structured set if the second is a homomorphic image of the first. They are abstractly identical if each is an isomorphic image of the other. Now if no mapping from one structured set into another structured set can be a homomorphism then the second set is said to be anbomomorpbic to the first set. If no mapping from one structured set into another structured set can be an isomorphism then the second set is said to be anisomorpbic to the first set. 
Results: Lemma: If a structured set is anbomorpbic to a given structured set then it is anisomorphic to the given structured set.

Proof. By reductio ad absurdum; if there exists an isomorphism then there exists a homomorphism. But that condition contradicts the hypothesis concerning the anhomomorphic nature of the image.

The following theorem draws on previous results dealing with the theory of mutant sets, [1], [2], in order to establish that, in a very general sense, there exists a pair of structured sets for which one is an anhomomorphic image of the other.

Theorem: Let $(A, *)$ and $(B, 0)$ be two algebraic systems that satisfy left-associative (right-associative or both) binary composition laws when three or more elements are composed. Let $M$ be a $(\lambda, S)$-mutant set of $(B, 0)$. Then there is no bomomorphism from the $T$ of a subsystem $(T, *)$ of $(A, *)$ into (i.e., onto a subset of) $M$.

Proof. Put $(A, *)$ and $(B, \mathrm{o})$ equal to the additive group of all integers. Put $M$ equal to the set of all odd integers. Put $T$ equal to the set of all even integers. Clearly there is a one-one mapping from $T$ onto $M$. Hence the theorem is not vacuously satisfied. Now if $M$ is empty there is no mapping and, a fortiori, no homomorphism. Hence suppose $M$ is nonempty. If there exists such a homomorphism $\phi$, i.e., if there exists an into mapping $\phi: T \rightarrow M$ which for every $a \epsilon T$ and $b \epsilon T$ satisfies $\phi\left(a^{*} b\right)=$ $\phi(a) o \phi(b)$, then for $t_{i} \in \mathrm{T}$ and, e.g., with a left-associative binary composition law assumed, if $\lambda \geq 3, \phi\left(t_{1} * \ldots * t_{\lambda}\right)=\phi\left(t_{1}\right) \mathbf{o} \ldots$ o $\phi\left(t_{\lambda}\right) \epsilon \bar{M} \cap S \subseteq B$. Since $T$ is closed, $t_{1} * \ldots * t_{\lambda} \epsilon T$. Hence $\phi\left(t_{1} * \ldots * t_{\lambda}\right) \in M \subseteq B$; a contradiction. Note that the proof is intuitionistically satisfactory in the sense that the law of excluded-middle is not used.

Corollary: Under the conditions of the theorem, $M$ is anbomomorphic to $T$ and, a fortiori, $M$ is anisomorphic to $T$.

This research was sponsored, in part, by the NATIONAL SCIENCF FOUNDATION and GENERAL DYNAMICS CORPORATION.

\section{REFERENCES}

[1] A. A. Mullin, "Some Remarks on a Relative Anti-closure Property," Zeit. math. Logik Grundlagen Math., vol. 7, No. 2 (June, 1961) pp. 99-103; erratum: in Theorem 1.7 and Corollary 1.3, the mutant set $M$ should be nonempty, in general. 
160 ON DIFFERENCES OF CERTAIN STRUCTURED SETS

[2] A. A. Mullin, "Some Theorems on the Structure of Mutant Sets and their Applications to Group and Ring Theories," Notre Dame Journal of Formal Logic, vol. III, No. 3 (July, 1962), pp. 148-151.

University of Illinois

Urbana, Illinois 